\title{
Design of a fuel-efficient two-stroke Diesel engine for medium passenger cars: comparison between standard and reverse uniflow scavenging architectures
}

\author{
Jeremy Galpin ${ }^{1,2}$, Thierry Colliou ${ }^{1,2}$, Olivier Laget ${ }^{1,2}$, Fabien Rabeau ${ }^{1,2}$, Gaetano De Paola ${ }^{1,2}$, Pascal Rahir ${ }^{3}$ \\ 1 IFP Energies nouvelles \\ 2 Institut Carnot IFPEN Transports Energie \\ 3 Groupe Renault
}

\begin{abstract}
In spite of the increasingly stringent emission standards, the constant growth of road traffic contributes to climate change and induces detrimental effects on the environment. The European REWARD project (REal World Advanced Technologies foR Diesel Engines) aims to develop a new generation of Diesel engines complying with stricter post Euro 6 legislation and with lower $\mathrm{CO}_{2}$ emissions. Among the different technologies developed, a fuel-efficient two-stroke Diesel engine suited for $\mathrm{C}$-segment passenger cars will be designed and experimentally evaluated.

One major challenge for two-stroke engines is the achievement of an efficient scavenging. As the emptying of the in-cylinder burnt gases and the filling by fresh gases is performed at the same time, the challenge consists in removing as much burnt gases as possible while avoiding the by-pass of fresh air toward the exhaust line. For the considered application, the uniflow scavenging architecture, which is featured by ports located in the bottom of the cylinder and valves in the head, is selected. Two possible arrangements for the intake and the exhaust are compared: either the standard configuration for which the intake is ensured by the ports and the exhaust by the valves or the reverse configuration.

Both standard and reverse configurations are first compared through 0D system simulations, performed with LMS Imagine.Lab Amesim and then by 3D CFD simulations with CONVERGE. The standard configuration is favored, thanks to better ISFC performances, especially at low and medium loads operating conditions. In addition, the scavenging is strongly penalized for the reverse architecture due to the drag downstream the intake valves.
\end{abstract}

\section{Introduction}

The fight against the global warming and the reduction of the atmospheric pollution are meaningful environmental concerns in our industrial societies and involve pursuing the continual effort of reduction of the fuel consumption and of control of emissions induced by internal combustion engines. The REWARD collaborative project (REal World Advanced Technologies foR Diesel Engines), funded by the European Union within the Horizon 2020 framework, takes part to this technical challenge and aims more specifically to develop a new generation of Diesel engine even more

Page 1 of 12 efficient and complying with future stringent regulations of pollutant emissions. Among the different technologies developed, a fuelefficient two-stroke Diesel engine suited for C-segment passenger cars is designed and experimentally evaluated; the partners involved in these activities are: Groupe Renault, CMT-Universitat Politècnica de València, Czech Technical univ., Delphi and IFP Energies Nouvelles.

Two-stroke Diesel engines offer high power density, associated with a low weight and a small engine size. These particular qualities are especially well suited for Marine engines, which can reach very high thermal efficiency due among others to the very low rated speed of the engine (about 100RPM) and the large stroke-to-bore ratio (up to 4).

Regarding on-road light duty applications, four-stroke engines represent almost the entire market of Diesel applications even if a renewed interest for two-stroke Diesel engine has recently come up. The development of two-stroke engine suited for passenger cars is motivated by the reduction of the mass, the bulk and the cost of the powertrain thanks to the double combustion frequency: the number of cylinders can be reduced with a very low impact on the $\mathrm{NVH}$ and maintaining a high unitary displacement and thus high combustion efficiency. In addition to those advantages, two-stroke engines have also the potential of reducing engine-out NOx emissions, as pointed out by Xin [1], thanks to the natural operation of two-stroke engine with large amount of residuals gases. Indeed, this property is particularly interesting for transients because the latency inherent to a classical Exhaust Gas Recirculation system is avoided and lower NOx emissions can be thus anticipated. Moreover, Warey et al. [2] have underlined that two-stroke Diesel engine may also yield a benefit in terms of $\mathrm{CO}_{2}$ emissions. Indeed, Warey et al. have compared, on the basis of system simulations, two concepts of twostroke engine and an hypothetical state-of-the art four-stroke Diesel engine in 2020. Their results show that the $\mathrm{CO}_{2}$ emissions over two normalized driving cycles are at least reduced by $5 \%$. In this frame, several attempts have been done to develop an efficient two-stroke engine suited for passenger cars. The works of Knoll [3], Masuda et al. [4], Nomura and Nakamura [5], Tribotté et al. [6], Brynych et al. [7] and Redon et al. [8] may especially be mentioned.

The basic difference between two-stroke and four-stroke engines is that the combustion occurs each revolution. This special feature implies that the transfers of gases around the bottom dead center (BDC) have to be performed within a small available time and the 
technical solution consists in almost fully overlapping the phases of exhaust of burnt gases and of intake of fresh gases. This gases transfer process is called scavenging, and has to be well optimized because it strongly affects the overall efficiency of the engine (reduced volumetric efficiency due to a poor removal of residual gases). The main challenge consists in trapping a maximal amount of fresh gases while removing as much as possible burnt gases and avoiding at the same time, the waste of fresh gases by a direct bypass to the exhaust. From a local point of view, this involves that the fresh gases may push the in-cylinder burnt gases toward the exhaust without mixing, and that the flow is stopped once all the burnt gases have exited the cylinder. Therefore a perfect scavenging is basically featured by no mixing and an excellent timing.

Several engine architectures, described by Schweitzer [9] and listed here below, were developed in order to produce this ideal process:

- Engines with both intake and exhaust ports, without camshafts, which strongly reduces engine friction losses and presents the interest of a simplified mechanical layout. Two main scavenging configurations are available according to the arrangement of the ports around the cylinder. The first solution consists in setting the ports in opposite sides of the cylinder. This configuration called cross-flow scavenging is generally implemented with a deflector on the piston in order to compel the fresh gases to flow toward the top and thus scavenge the burnt gases from the top of the cylinder. The second arrangement is the loop scavenging configuration, with the ports located on the same side of the cylinder. This particular distribution of the ports aims to impel a U-turn movement of the flow into the cylinder. However, the proximity of the intake and exhaust facilitates the short-circuiting of fresh gases: this issue may be solved through a detailed optimization and design of the intake ports. Mattarelli et al. [10] performed such optimization on the basis of 1352 CFD simulations, related to different transfer ports inclinations and different operating points. Lastly, one may mention opposed piston engines which also operate with ports only. These engines may reach a very efficient scavenging [11], but the management of a very long stroke and of two crankshafts introduce a noticeable difficulty, especially for light-duty applications which require simple and compact solutions.

- The poppet valves engines enable the loop scavenging. The main advantage of these engines is the mechanical configuration, very close to a conventional four-stroke engine. Despite this advantage, the loop flow motion make the development of a proper swirl motion very difficult and the associated combustion system has to deal with this constraint. Moreover, Ternel et al. [12], who developed a twin cylinder poppet valve engine, also emphasize that the design of the air loop is a prior parameter for achieving an efficient scavenging. Finally, permeability of valves is low compared to ports; this implies larger differential pressures between the intake and exhaust, which strongly penalizes the engine fuel consumption due to the energy required by an additional mechanical compressor.

- An intermediate architecture involves both ports and valves. The associated scavenging is called uniflow because of the induced main axial flow motion in the cylinder. Abthoff et al. [13] have benchmarked fully-valves and uniflow scavenged engines dedicated to passenger cars application. Their results show that lower fuel consumption and larger specific power are achievable with the uniflow engine. The main reasons are a best fitted in-cylinder aerodynamics for combustion and a more efficient scavenging. Laget et al. [14] have also shown by Computational Fluid Dynamics (CFD) simulations that an efficient scavenging can be reached with the uniflow scavenging if the ports are properly designed. In the frame of two-stroke gasoline engines, Ma et al. [15] have also optimized by CFD the ports of an uniflow scavenged engine and the CFD outputs have been implemented in a system code model for assessing the engine performances.

Due to its better efficiency mentioned by Abthoff et al. [13], the uniflow scavenging architecture has been selected for this project, and two possible arrangements are analyzed: either the standard uniflow configuration with an the intake ensured by ports and the exhaust by poppet valves or the reverse configuration.

Both standard and reverse uniflow configurations are benchmarked on the basis of system simulations, performed with LMS Imagine.Lab Amesim, and 3D CFD simulations, performed with CONVERGE version 2.2. System simulations were used to assess the specific fuel consumptions for several operating points, by assuming hypotheses such as a similar scavenging. 3D CFD simulations allow detailed representation of the scavenging process but, due to longer computational time, it was used only for a single operating point with given exhaust and intake diagrams.

The benchmark of the uniflow configurations is performed on the basis of a single cylinder engine, whose properties are detailed in the first part. System simulation results are then discussed and the last part is devoted to the analysis of the CFD results before drawing some conclusions.

\section{Engine architecture}

The features of the studied engine are listed in Table 1 . The bore and stroke are respectively equal to $76 \mathrm{~mm}$ and $88 \mathrm{~mm}$, and are related to the Renault K9K engine which is a four-stroke Diesel engine with a capacity of 1.46 liters. Even if a single cylinder engine architecture is here studied, the addition of one or two cylinders may be achieved to reach the targeted power for the considered C-segment passenger cars application with a specific power of $60 \mathrm{~kW} / \mathrm{l}$. A supercharger is used in addition to the turbocharger because of the necessity to ensure a net positive differential pressure between the intake and the exhaust on the whole engine map. The fuel is introduced in the cylinder by a direct injection system and the injector is located on the axis of the combustion chamber and at the center of the cylinder head where four poppet valves are implemented.

Table 1. Features of the engine configuration

\begin{tabular}{|l|l|}
\hline Displaced volume & $400 \mathrm{~cm}^{3}$ \\
\hline Stroke & $76 \mathrm{~mm}$ \\
\hline Bore & $88 \mathrm{~mm}$ \\
\hline Connecting Rod & $180 \mathrm{~mm}$ \\
\hline Geometrical compression ratio & 16 \\
\hline Numbers of valves / ports & $4 / 12$ \\
\hline Supercharging & Turbocharger and supercharger \\
\hline
\end{tabular}

Page 2 of 12 


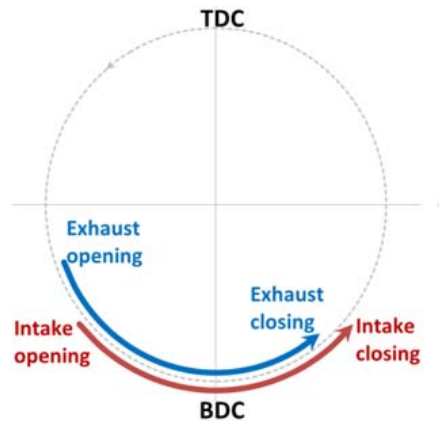

(a) Standard scavenging - intake with ports and exhaust with valves

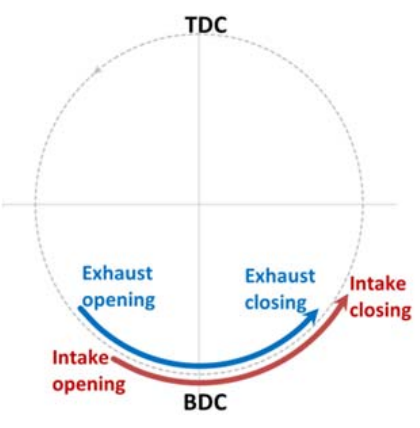

(b) Reverse scavenging - intake with valves and exhaust with ports
Figure 1. Schematic representation of the intake and exhaust diagrams and comparison between the standard and reverse uniflow scavenging

\section{System simulation analysis}

The standard and reverse uniflow configurations are benchmarked by LMS Imagine.Lab Amesim system simulation code. This code is used by worldwide automotive OEMs and suppliers to predict the multidisciplinary performance, efficiency and emissions of their systems. One of the main differences between the standard and reverse configurations is the timing of the intake and exhaust processes. First, it has to be mentioned that even if the ports generally offer larger permeability than valves, their diagram is hardly flexible and symmetric around the bottom dead center as the opening and closing is directly controlled by the piston motion. An offset of the piston wrist pin may allow small extent but this possibility is not here studied by simplification.

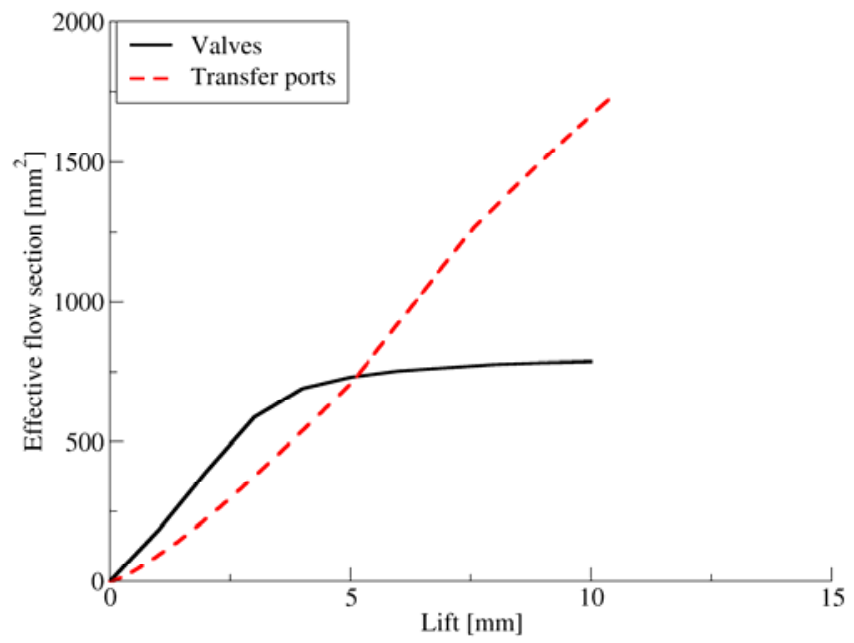

Figure 2. Effective flow section for the valves and the ports

Generally, the opening of the exhaust has to occur before the opening of the intake in order to allow a sufficient decrease of the in-cylinder pressure, which enables the entering of fresh gases into the cylinder and avoids the back-flow of burnt gases in the intake. Due to the constraint of symmetrical ports diagrams, the standard uniflow configuration is featured by an early opening of the exhaust relative to the bottom dead center which induces a truncated expansion stroke. On the contrary, the reverse configuration involves a late closing of the intake which truncates the compression stroke. The reduction of the expansion and compression phases respectively for the standard and reverse configuration are illustrated in Figure 1. The balance between a shorten expansion and a shorten compression is here discussed on the basis of fuel consumptions assessed by system simulations.

\section{Hypotheses}

A system model of the engine described above was developed in LMS Imagine.Lab Amesim. Some hypotheses were considered for the simulations:

- The combustion is modeled by the Dual Flame Model (DFM) from Rudloff et al. [16] available in the IFP-engine library. This model introduces a phenomenological representation of the combustion and accounts for the premixed and the mixing controlled phases of the combustion. The CA50 is kept constant to 10 CAD after TDC for all cases.

- The same set of parameters for the combustion model is used for both uniflow configurations, whereas they might differ on the in-cylinder aerodynamic.

- No EGR (exhaust gas recirculation) is considered, for simplification, and the engine is fed by air only.

- Acoustics effects are not taken into account since the air loop including the turbocharger, supercharger, intercooler and connecting pipes is not explicitly represented.

- One cylinder is modeled, possible interferences among cylinders are thus neglected. The hypothesis does not bring a gap from reality because the final design of the engine will intend to limit cylinders interferences as they might alter the engine performances.

- The pressures $P_{2}$ and $P_{3}$, respectively the intake and the exhaust pressures, are imposed as discussed below. The temperature at the intake $T_{2}$ is set to $313 \mathrm{~K}$.

- The opening of the ports is controlled by the flat part of the piston and the early opening induced by the first ring is neglected.

- The effective flow section related to the four valves and the transfer ports are displayed in Figure 2. From a global point of view, the ports offer a significant flow section compared to the valves as mentioned above. The effective flow section for the valves tangentially reaches a maximal value of $800 \mathrm{~mm}^{2}$, which indicates that the minimal flow section is shifted in the duct for large lifts. Regarding the ports, the effective flow section linearly varies with the uncovering of the ports. This indicates that the effective section is close to the geometrical section. Finally, one may underline that the effective sections do not take into account the direction of the flow in the system simulations, i.e. the minimal flow section is assumed to be the same whatever the flow direction.

Page 3 of 12 


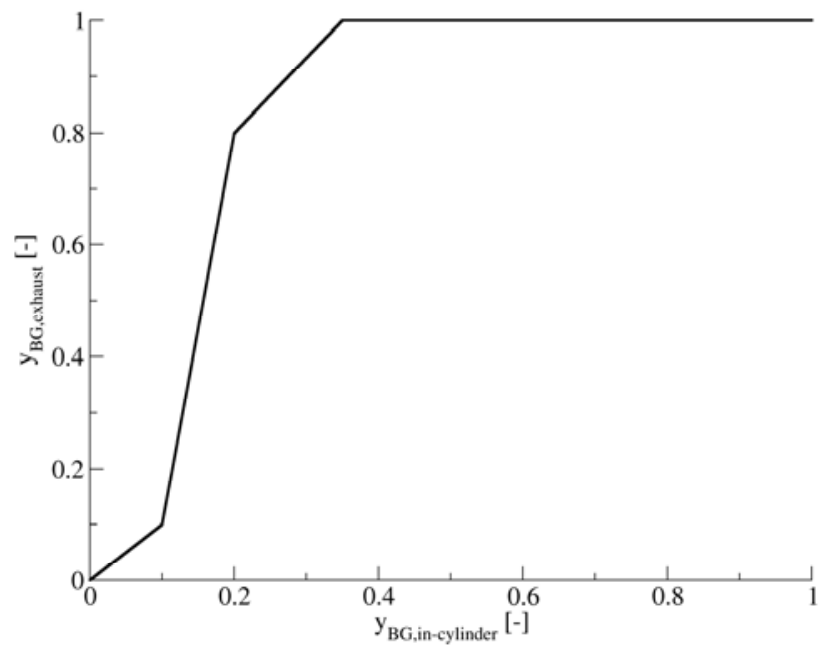

Figure 3. Scavenging curves implemented in the system simulations

A dedicated module for the scavenging has been implemented with an imposed scavenging curve as displayed in Figure 3. The scavenging curve is needed in order to characterize the efficiency of the scavenging, as it represents the mass fraction of burnt gases found in the cylinder versus the one observed in the exhaust. The starting point of the curve at the opening of the exhaust is the top right, and it moves toward the left until it reaches the bottom left at the end of the scavenging. Regarding the system simulation code, the same curve, plotted in Figure 3, is used for all the operating points and for both standard and reverse configurations. This curve is not representative of an actual geometry computed using 3D CFD or experimentally measured, but it corresponds to a targeted scavenging process.

\section{Methodology}

Three operating conditions representative of the targeted application are investigated: $1500 \mathrm{rpm} \times 4$ bar IMEP (low speed and low load), 2000 rpm x 7 bar IMEP (medium speed and medium load) and 3000 rpm x 11 bar IMEP (high speed, full load). For each operating point and uniflow configuration, a design of experiments (DOE) is computed with the following variable parameters:

- The engine differential pressure $\Delta P=P_{2}-P_{3}$ is equal to 200 mbar for the $1500 \mathrm{rpm} \times 4$ bar and 400 mbar for the two other operating points, as reported in Table 2. These values came from the experience on previous studies [6-7].

- The investigated ranges of intake pressure $P_{2}$ are given in Table 2 and depend on the operating point. The studied ranges of pressure allows in reaching fuel air equivalence ratios similar to conventional four-stroke Diesel engines.

- For both configurations, the maximal valve lift is set to about $6 \mathrm{~mm}$ and three diagrams for the valves with durations of 90, 100 and $110 \mathrm{CAD}$ are investigated.

- For the standard uniflow configuration, the investigated diagrams are reported in Table 3 . The duration of the piston controlled intake stroke varies from 80 to $110 \mathrm{CAD}$, and

Page 4 of 12 this is respectively related to an opening from 140 to 125 CAD after TDC. For the exhaust diagram, a sensitivity to the valve opening from 100 to 140 CAD is performed. Both intake and exhaust diagrams are plotted in Figure 4-a.

- Regarding the reverse configuration, a comprehensive study of the influence of the intake diagrams is performed by testing a variation of openings from 130 to 170 CAD. Durations from 100 to 140 CAD for the piston controlled exhaust stroke are investigated: the resulting exhaust opening varies from 130 to 110 CAD after TDC. Figure 4-b shows the studied intake and exhaust diagrams.

Table 2. Investigated operating conditions

\begin{tabular}{|c|c|c|}
\cline { 2 - 3 } \multicolumn{1}{c|}{} & $\Delta P$ & $P_{2}$ \\
\hline $1500 \mathrm{rpm} 4 \mathrm{bar}$ & $200 \mathrm{mbar}$ & $1300-2800 \mathrm{mbar}$ \\
\hline $2000 \mathrm{rpm} 7 \mathrm{bar}$ & $400 \mathrm{mbar}$ & $1400-2300 \mathrm{mbar}$ \\
\hline $3000 \mathrm{rpm} 11 \mathrm{bar}$ & $400 \mathrm{mbar}$ & $2500-3800 \mathrm{mbar}$ \\
\hline
\end{tabular}

The system computations provide a direct assessment of the ISFC related to the high-pressure loop of the $\mathrm{P}-\mathrm{V}$ diagram. A corrected ISFC is also defined in order to take into account the supercharger work which is withdrawn from the engine work. Constant turbine and compressor efficiencies are used, respectively equal to 0.7 and 0.6 , and the supercharger efficiency is assumed to be equal to 0.65 . These global efficiencies are related to realistic values achievable with onmarket technologies such as the ones mentioned in [17]. Finally, the after-treatment system is modeled by a global pressure drop, and the back-pressure downstream is assumed to be the atmospheric pressure of 1.0bar. From a methodology point of view, the compression work necessary for reaching the targeted $\mathrm{P}_{2}$ is computed with the above mentioned hypotheses. The work of the compressor driven by the turbine is then subtracted and the resulting difference corresponds to the work of the supercharger. The latter is drawn off from the engine work in order to compute the corrected ISFC.

Corrected ISFC is assessed for each point of the DOE mentioned above, and abnormal points with too large fuel consumption are removed from the data-base results.

Table 3. Investigated intake and exhaust diagrams for the standard and reverse uniflow configurations

\begin{tabular}{|c|c|c|c|}
\cline { 3 - 4 } \multicolumn{2}{c|}{} & Standard conf. & Reverse conf. \\
\hline Int. duration & CAD & $80,90,100,110$ & $90,100,110$ \\
\hline Int. opening & CAD af. TDC & $140,135,130,125$ & $130,140,150,160,170$ \\
\hline Exh. duration & CAD & $90,100,110$ & $100,120,130,140$ \\
\hline Exh. opening & CAD af. TDC & $100,120,130,140$ & $110,115,120,130$ \\
\hline
\end{tabular}




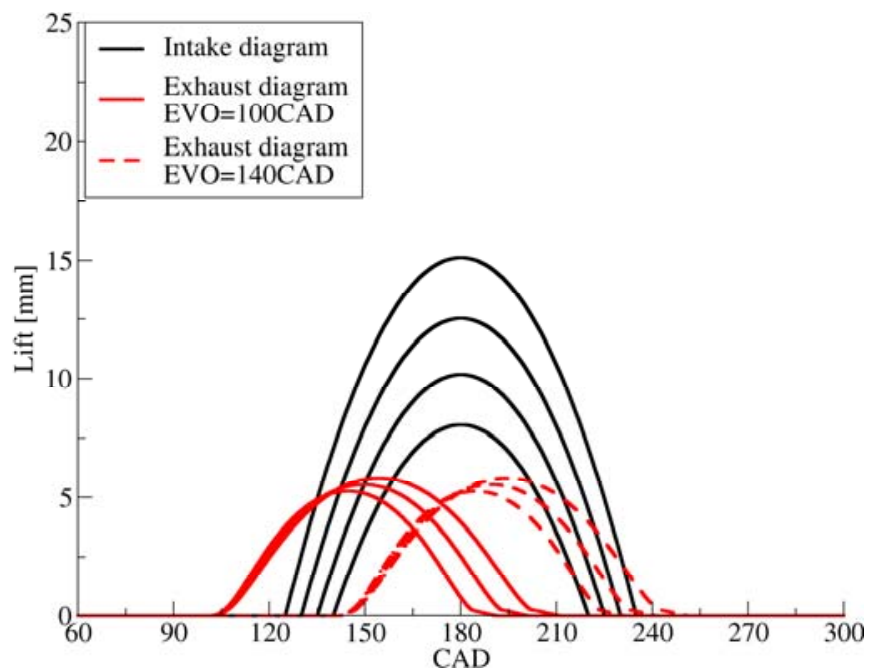

(a) Standard configuration

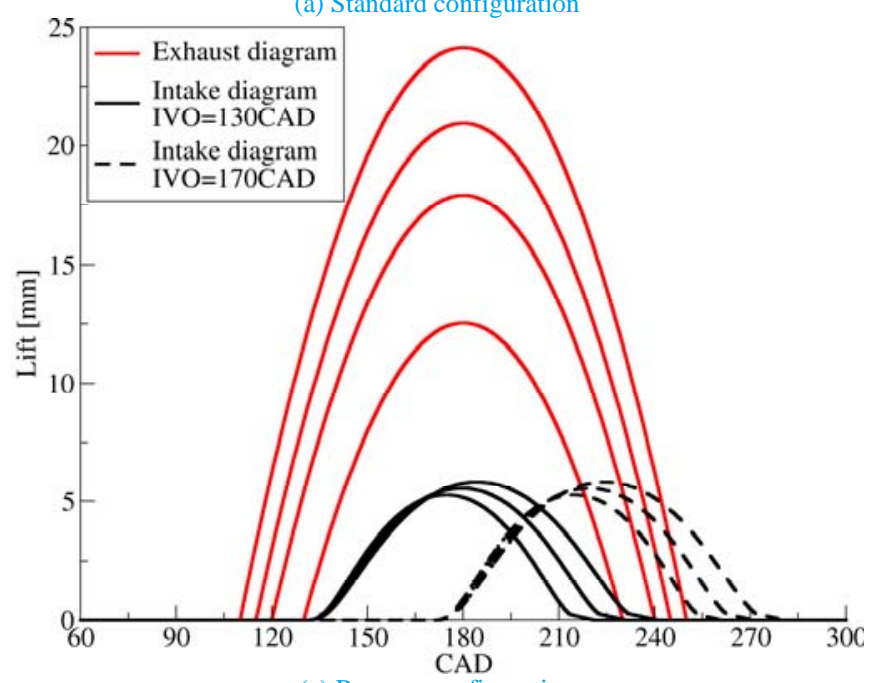

(a) Reverse configuration

Figure 4. Temporal evolutions of the intake and exhaust diagrams for the standard and reverse uniflow scavenging

\section{Corrected ISFC results}

The evolution of the corrected ISFC versus the intake pressure is showed in Figure 5-a for the 1500 rpm x 4 bar operating point. It may be pointed out that the corrected ISFC increases with the intake pressure for both configurations, due to the energy required by the supercharger. This suggests that a minimal intake pressure should be preferred for this operating condition, in order to minimize the fuel consumption. Corrected ISFC for both configurations are very similar, nevertheless the lowest ISFC is obtained using the standard configuration (around $3 \mathrm{~g} / \mathrm{kWh}$ lower).

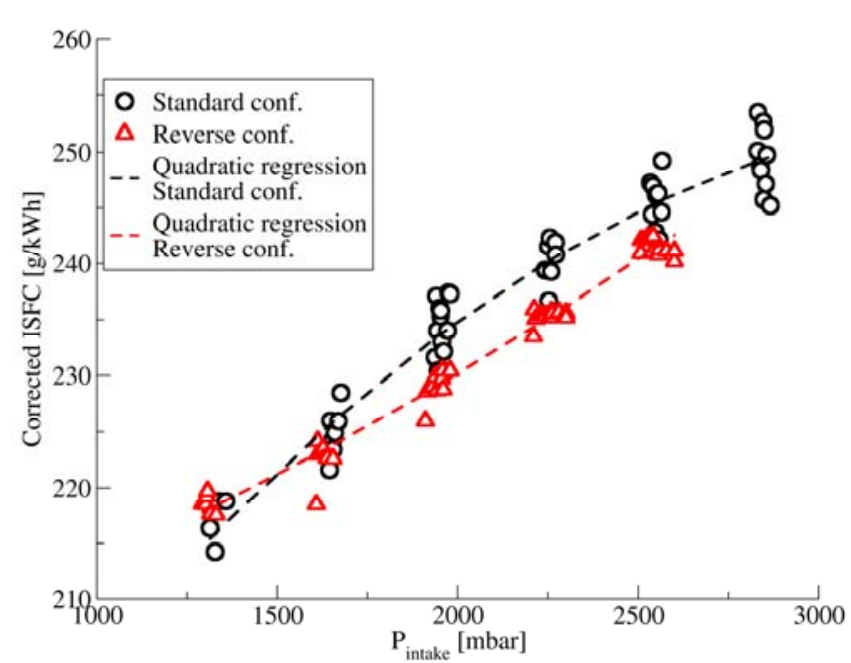

(a) $1500 \mathrm{rpm} \times 4$ bar

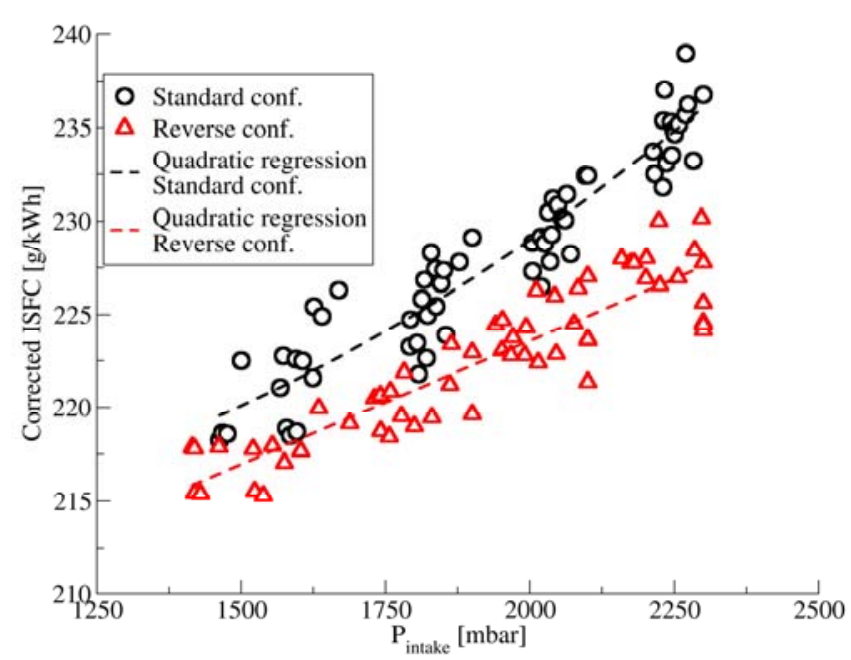

(b) $2000 \mathrm{rpm} \times 7$ bar

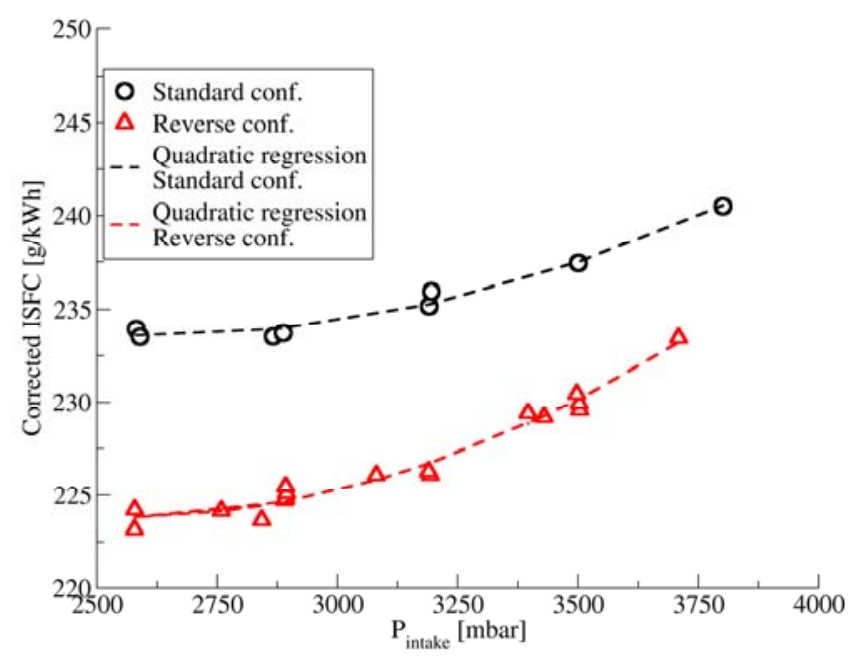

(c) $3000 \mathrm{rpm} \times 11$ bar

Figure 5. Corrected ISFC vs. intake pressure for three operating points and comparison between the standard and reverse configurations. 
The same analysis is performed at the $2000 \mathrm{rpm}$ x 7 bar as shown in Figure 5-b. From a global point of view, the corrected ISFC increases with the intake pressure for both configurations as pointed out for the $1500 \mathrm{rpm} \times 4$ bar operating point. A slight benefit, smaller than 5 $\mathrm{g} / \mathrm{kWh}$, is here provided by the reverse configuration for an intake pressure larger than 1600mbar.

The full load case is investigated in Figure 5-c. The results show again an increase of the corrected ISFC with the intake pressure, but the slope is smaller compared to the other operating points. The comparison between the standard and reverse configurations shows that there is a significant benefit for the reverse configuration for any level of intake pressure. When looking at the lowest intake pressure, the difference in terms of fuel consumption is about $10 \mathrm{~g} / \mathrm{kWh}$. From those results it may be concluded that the reverse uniflow configuration presents lower fuel consumption, but this advantage appears only for the full load.

\section{Analysis of the trapped masses}

Figure 6 investigates the evolution of the charging ratio versus the ratio between expansion and compression ratio for the three studied operating points. The charging ratio [9] quantifies the capability of the engine in trapping fresh gases. It is defined as the ratio between the trapped mass of air after the intake closure and a reference mass which depends on the intake conditions:

$C R=m_{F G, \text { trapped }} / m_{F G, \text { reference }}$

Where $m_{F G \text {,trapped }}$ is the mass of trapped fresh gases and $m_{F G \text {,reference }}$ is a reference mass of fresh gases expressed as:

$m_{F G, \text { reference }}=\frac{P_{2}}{r \cdot T_{2}} \cdot V_{\text {sweep }}$

where $r=287 \mathrm{~J} \cdot \mathrm{kg}^{-1} \cdot \mathrm{K}^{-1}$ is related to air and $V_{\text {sweep }}$ is the engine capacity.

The expansion to compression ratio is defined as the ratio of the incylinder volume at exhaust opening (EO) compared to the volume at intake closure (IC):

$E C R=V_{c y l i n d e r, E O} / V_{c y l i n d e r, I C}$

When comparing both uniflow configurations, it may be pointed out that the reverse configuration yields smaller charging ratios. The analysis of the maximum values, shown by cross symbols, indicates that the charging ratios for the reverse configuration are decreased by about $7 \%$ for the $1500 \mathrm{rpm} \times 4$ bar and $2000 \mathrm{rpm} \times 7 \mathrm{bar}$ operating points, and only $3 \%$ for the $3000 \mathrm{rpm} \times 11$ bar operating point. This reduction is due to the shift of the scavenging toward the compression side of the cycle. Indeed, at the end of the process, the rise of the piston induces an in-cylinder pressure increase which prevents the fresh air from entering in the cylinder or, in the worse situations, induces a back-flow from the cylinder to the intake plenum. Despite this, the disadvantage of the reverse configuration pointed out on the charging ratio almost vanishes for the full load operating point, since the penalty is divided by two. Regarding the expansion to compression ratio, a longer expansion is obtained for all the operating points for the reverse configuration. The observed difference of maximum values of charging ratio (indicated by cross symbols in Figure 6) is about $30-40 \%$. This benefit is the consequence of the delayed opening of the exhaust, as discussed above. As a consequence, there is a competition between the benefit of a longer expansion and the limitation of the amount of trapped fresh air. This tradeoff depends on the operating point. For the 3000 rpm x 11 bar operating point, the loss of charging ratio is small (-3\%) compared to the increase of the expansion to compression ratio. Moreover, the in-cylinder pressure is still large at the end of the expansion for enabling an important additional work through the longer expansion. For the other two operating points, the additional expansion is associated to a decrease of the amount of trapped air, and results poorly exploited due to the low pressure remaining in the cylinder at the end of the expansion.

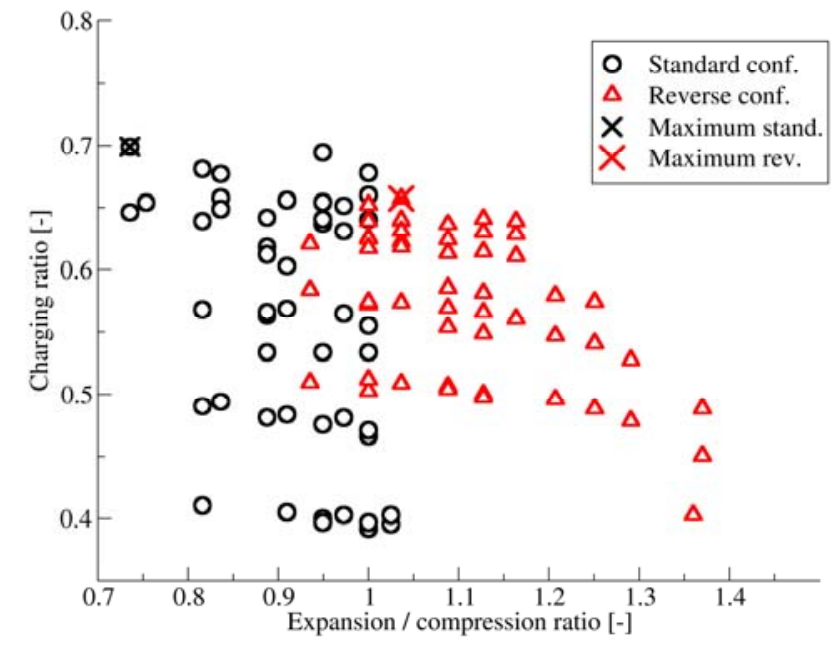

(a) $1500 \mathrm{rpm} \times 4$ bar

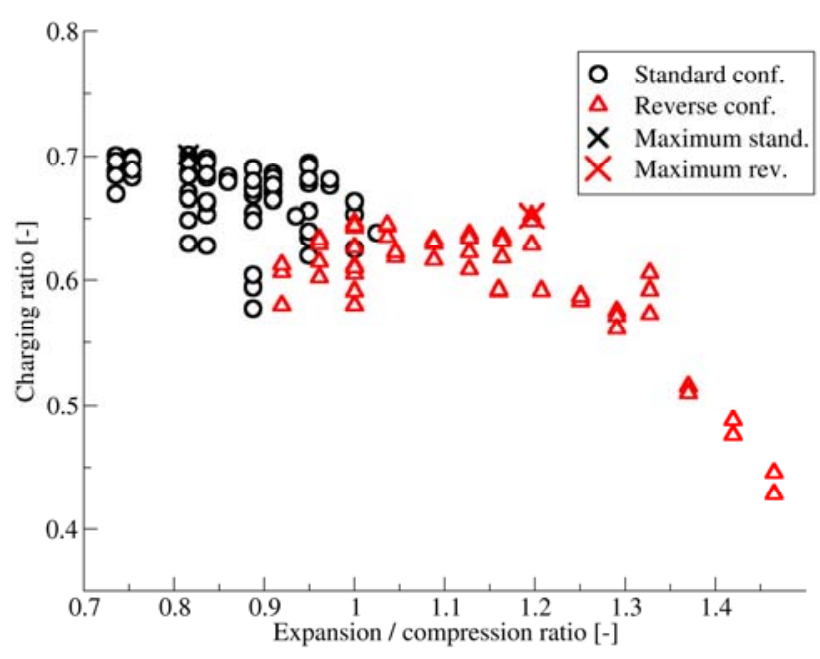

(b) 2000 rpm x 7 bar

Page 6 of 12 


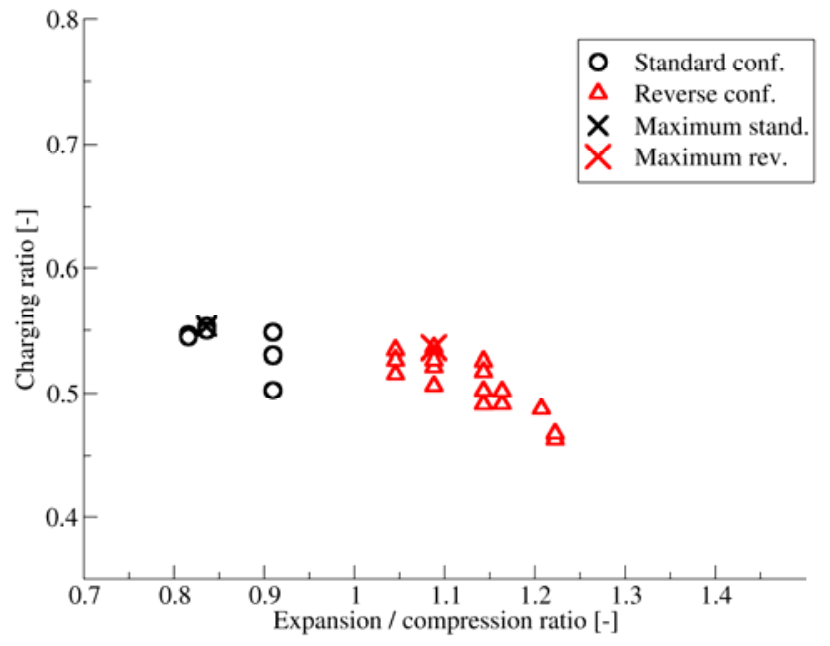

(c) $3000 \mathrm{rpm} \times 11$ bar

Figure 6. Charging ratio vs. the expansion to compression ratio for three operating points and comparison between the standard and reverse configurations.

To conclude, the analysis of the specific fuel consumption differences, through the study of charging ratio and expansion to compression ratio, points out the differences between mid-medium and high load operating conditions. The reverse configuration is here more efficient for the full load because the additional expansion occurs with a small penalty of the charging ratio. However, for the other operating points, both effects are balanced and the reverse configuration does not show any clear advantage. It may also be reminded that these conclusions are based on the assumption that the scavenging is equal for both uniflow configurations. The following section focuses on the analysis and assessment of this hypothesis.

\section{D CFD analysis}

A first evident difference between the scavenging occurring in the standard and reverse uniflow configurations is the flow orientation. In the standard architecture, the flow is oriented from the bottom to the top of the cylinder, whereas for the reverse it goes from the top to the bottom. Another significant difference is the way to admit fresh gases into the cylinder: for the standard configuration, the flow comes from the bottom of the cylinder through ports, while in the reverse configuration the fresh air comes through valve curtains which may represent a restriction for the flow. In order to assess the consequences of these differences on the scavenging itself, 3D CFD simulations were performed using the CFD software CONVERGE version 2.2 [18] on the $3000 \mathrm{rpm} \times 11$ bar operating point.

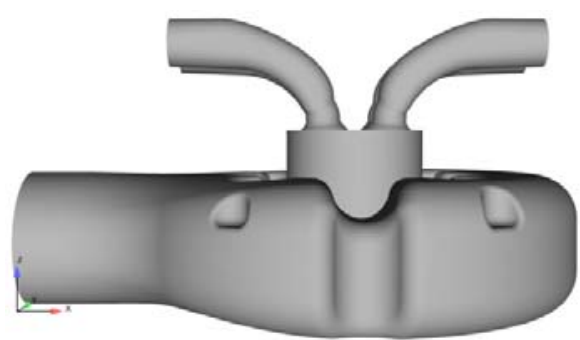

(a) Case 1 - standard conf.
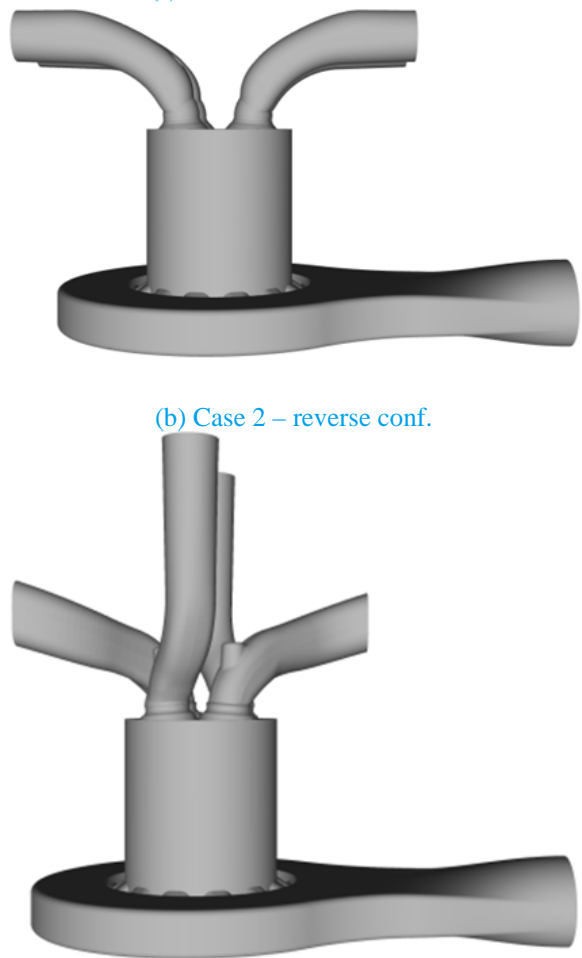

(c) Case 3 - reverse conf.

Figure 7. Investigated geometries by CFD.

\section{Investigated cases}

Three cases related to the three geometries shown in Figure 7 were investigated. Case 1 is devoted to the standard configuration, its geometry is displayed in Figure 7-a. The intake is performed by 12 ports and is fed by an intake plenum with a volume ensuring an almost constant intake pressure. The exhaust is controlled by 4 valves corresponding to 4 separated ducts. Cases 2 and 3 are devoted to the reverse configurations. Their geometries shown respectively in Figure 7-b and c differ by the intake ducts. The intake pipes of Case 2 are spark-ignition engine like ducts and mainly promote the filling without generating aerodynamic swirl motion. Regarding Case 3, the ducts are inspired from conventional four-stroke Diesel engine. Two tangential and two helicoidal ducts are arranged in order to impel a coherent in-cylinder swirl motion. It has to be highlighted that the plenum of Case 1 has larger volume than the ones of Cases 2 and 3, because of the lower density of intake gases with respect to exhaust gases.

The intake and exhaust diagrams and valve lifts for both configurations are presented in Table 4. They were chosen from 
previous works [14] and adapted to take into account the above mentioned system simulation results, for both standard and reverse configurations; i.e., the maximal lift is set to $6 \mathrm{~mm}$.

Table 4. Features of the investigated cases by CFD

\begin{tabular}{|c|c|c|c|}
\cline { 2 - 4 } \multicolumn{1}{c|}{} & & Standard conf. & Reverse conf. \\
\hline Intake duration & CAD & $125 \mathrm{CAD}$ & $100 \mathrm{CAD}$ \\
\hline Intake opening & CAD ATDC & $117 \mathrm{CAD}$ & $150 \mathrm{CAD}$ \\
\hline Exhaust duration & $\mathrm{CAD}$ & $95 \mathrm{CAD}$ & $120 \mathrm{CAD}$ \\
\hline Exhaust opening & CAD ATDC & $105 \mathrm{CAD}$ & $120 \mathrm{CAD}$ \\
\hline
\end{tabular}

\section{Numerical aspects}

CFD simulations address only the scavenging phase of the engine cycle. Only the period between the end of the expansion until the start of the compression is simulated. The initial conditions in terms of in-cylinder pressure, temperature and gas composition were provided from system simulations. For all the cases, a differential engine pressure of 0.3 bar is fixed, while the intake pressure $P_{2}$ is set to 2.8 bar.

CONVERGE code offers an automatic grid generation, approach particularly well suited for handling moving complex geometries, typical of two-stroke engines with ports. The reference cell size is set to $4 \mathrm{~mm}$ and refinements up to a cell size of $0.5 \mathrm{~mm}$ are applied close to walls and in areas with large gradients of velocities.

The turbulence is modeled owing to the k- $\varepsilon$ RNG model [19] with default constants, and a standard law-of-the-wall is used as wall treatment. Walls are non-slip, hydraulically smooth and their temperatures are assumed to be constant. Regarding the numerical aspects, the $2^{\text {nd }}$ order upwind scheme is retained for all balance equations, and the iterative method is the PISO algorithm from Issa [20]. The time step is variable in the simulation and defined as the minimum over the computational domain between a convective CFL number constrained below unity and an acoustic CFL number below 50.

\section{Assessment of the scavenging efficiency}

The efficiency of the scavenging is assessed through non-dimensional parameters, such as the charging ratio introduced above which provides information about the amount of fresh gases trapped after the intake closure. The trapping ratio [9] quantifies the efficiency of the trapping of the intake gases and thus yields information about the amount of fresh gases short-circuited. The scavenging ratio [9] is the proportion of fresh gases with respect to the in-cylinder total mass at the intake closure, and quantifies the efficiency of the scavenging of in-cylinder burnt gases. The trapping and scavenging ratios are respectively given by the following equations:

$T R=m_{F G, \text { trapped }} / m_{F G, \text { int ake }}$

$S R=m_{F G, \text { trapped }} / m_{\text {trapped }}$
Where $m_{F G \text {,intake }}$ and $m_{\text {trapped }}$ are respectively the intake mass of fresh gases and the total in-cylinder mass of gases at the end of the scavenging.

Table 5. Trapping, scavenging ratios and swirl numbers assessed by CFD

\begin{tabular}{|c|c|c|c|}
\cline { 2 - 4 } \multicolumn{1}{c|}{} & Case 1 & Case 2 & Case 3 \\
\hline Trapping ratio (Eq. 4) & $91 \%$ & $82 \%$ & $68 \%$ \\
\hline Scavenging ratio (Eq. 5) & $88 \%$ & $83 \%$ & $77 \%$ \\
\hline Swirl number @ 280CAD & 1.0 & 0.2 & 2.4 \\
\hline
\end{tabular}

The results in terms of trapping, scavenging and swirl numbers are presented in Table 5 for the three investigated cases: it may be pointed out that the best values are reached for the Case 1 which is the standard uniflow configuration. Among both reverse configurations, Case 2 yields better scavenging results than Case 3 . This suggests that the swirl has a negative effect on the scavenging for this uniflow configuration. Regarding the swirl motion at the end of the scavenging process, the geometries related to the Cases 1 and 3 generate a swirl respectively of 1.0 and 2.4 , while there is almost no swirl for the Case 2.

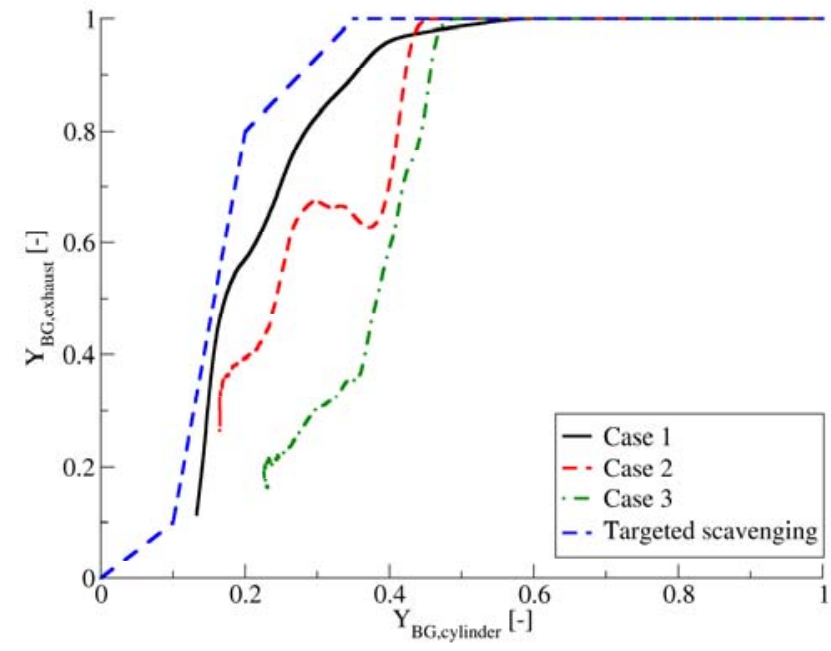

Figure 8. Scavenging curves computed by CFD.

The scavenging curves corresponding to the three studied cases are compared in Figure 8. The Case 1 provides the closest scavenging process to the target; the results related to the reverse configurations reveal less efficient scavenging processes: the scavenging curves drop quickly. The inflection points, for which the curves drop, are slightly moved toward smaller in-cylinder mass fraction of burnt gases for the reverse cases, in comparison to the standard case: this indicates that the by-pass of fresh air is slightly delayed. However, the decrease is stiff whereas the slope is lower for the Case 1 . Regarding Case 3, the decrease of the scavenging curve is monotone and declines quickly: this indicates that the short-circuiting of fresh gases occurs without removing burnt gases and with limited mixing with the burnt gases. For the Case 2, the decrease is not monotonic and a change in the slope of the curve can be observed for a mass fraction of burnt gases of about 0.3 . This break in the by-pass of fresh gases is characteristic of the passage of a pocket of burnt gases, and is

Page 8 of 12 
positive from a global point of view, as the scavenging curve is shifted toward lower in-cylinder mass fractions of burnt gases.

\section{Qualitative comparison}

In order to characterize the scavenging processes for the three studied cases, an in-cylinder tracer initialized to one in the cylinder and zero elsewhere is solved: Figure 9 shows a comparison of the tracer fields at different crank angles. It may be remarked that:

- The time 105CAD corresponds to the initial solution of the simulation with both the intake and exhaust closed.

- The discharge of the burnt gases can be seen at 115CAD for the standard Case 1 whereas it occurs later, around 135CAD for both reverse cases.

- $\quad$ For Case 1, the fresh gases enter into the cylinder via the top land at 135CAD, inducing an axial flow motion on the sides of the cylinder liner. At 155CAD, the in-cylinder charge is stratified, with burnt gases on the top and a mixing of fresh and burnt gases in the bottom. At the same time for both reverse cases, a backflow towards the intake manifold appears, due to a too low blow-down phase.

- The fresh gases reach the exhaust ducts at 175CAD for the Case 1. This leads to a drop of the scavenging curve, as discussed in the previous section. For both Cases 2 and 3, fresh gases enter into the cylinder and a vortex shedding develops downstream the valves.

- At $195 \mathrm{CAD}$, the scavenging is almost completed for the Case 1 whereas the process is still on-going for the reverse cases. The vortex shedding occurring downstream the valves leads to an extensive mixing between the fresh and burnt gases which is unfavorable to the scavenging process. For both geometries, fresh gases tend to flow preferentially along the cylinder liner which leads to a direct by-pass of fresh gases at the exhaust. When examining the difference between both reverse cases, it may be pointed out that burnt gases are trapped in the center of the cylinder for Case 3 because the intake ducts generate an intensive swirl and the small pressure area at the center prevents the burnt gases from exiting the cylinder. This behavior is not present in Case 2 because the intake ducts generate almost no swirl.

- $\quad$ For Case 3, the pocket of burnt gases trapped in the center of the cylinder can still be observed at $225 \mathrm{CAD}$ and is never removed from the cylinder. This explains the poor efficiency of the scavenging discussed in the previous section. For Case 2, a mixing of burnt and fresh gases still remains downstream the valves but the pocket of burnt gases trapped in the bottom of the cylinder still noticeable at 195CAD has been eventually cleared out. This explains the change in slope of the scavenging curve shown in Figure 8.

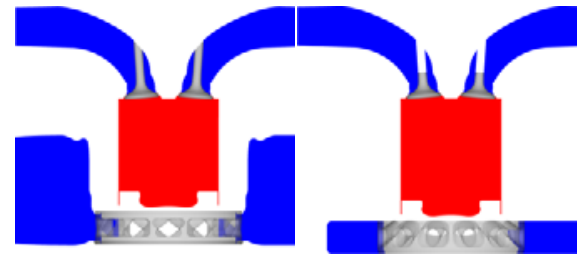

$105 C A D$

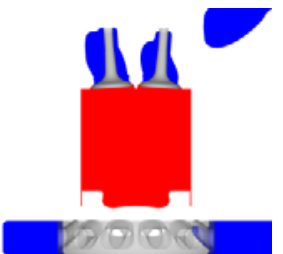

$105 C A D$
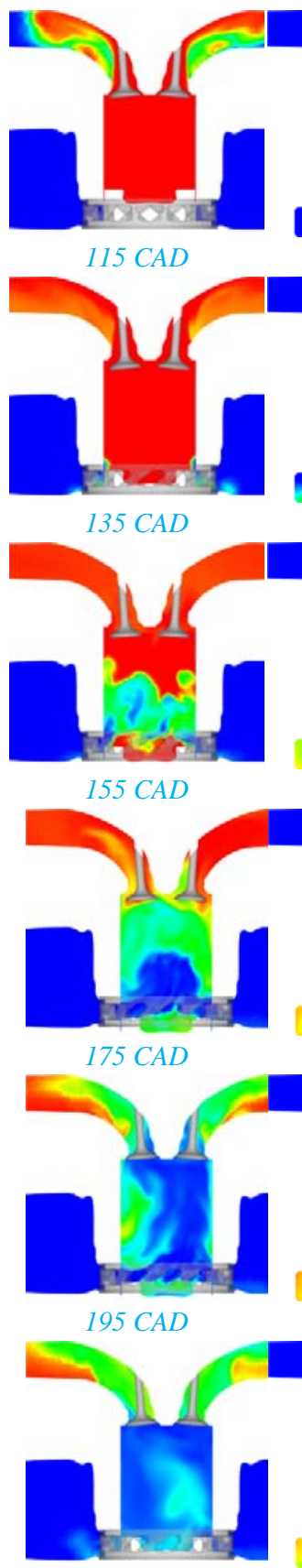

$225 C A D$
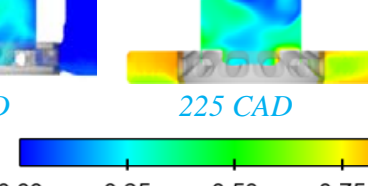
$115 C A D$
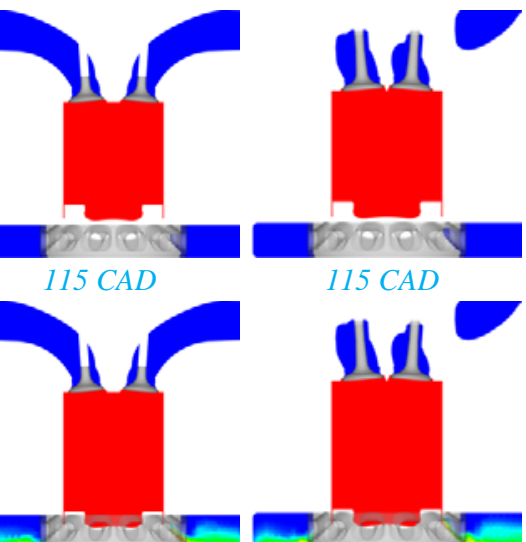

lik $135 C A D$
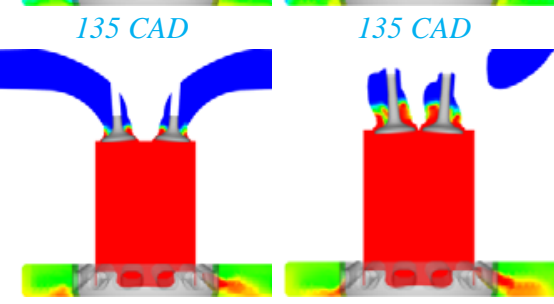

$155 C A D$

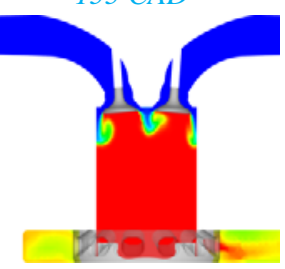

$155 C A D$

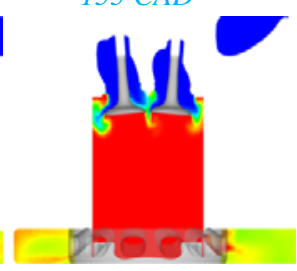

$175 C A D$

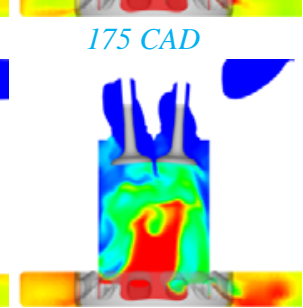

$195 C A D$

195 CAD

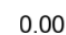

0.25

$225 C A D$

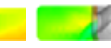

225 CAD

Figure 9. In-cylinder tracer fields (initialized at 1 in the cylinder et 0 elsewhere) and comparison between the three investigated cases.

In conclusion to this phenomenological analysis, the standard uniflow configuration allows getting the most efficient scavenging process among the different studied cases. The scavenging of the reverse configuration is penalized by the vortex shedding that develops downstream the valves. Moreover, the generation of swirl diminishes the scavenging, as it traps the burnt gases into the center of the cylinder and makes easier the by-pass of fresh gases. Setting apart the scavenging issue, this feature is also a constraint for the combustion 
system. Indeed, conventional combustion system generally operates with swirl number in the range of 1-2. As the swirl is almost nil for Case 2, a conventional system cannot be reemployed, and a dedicated combustion system compatible to zero in-cylinder swirl should be developed.

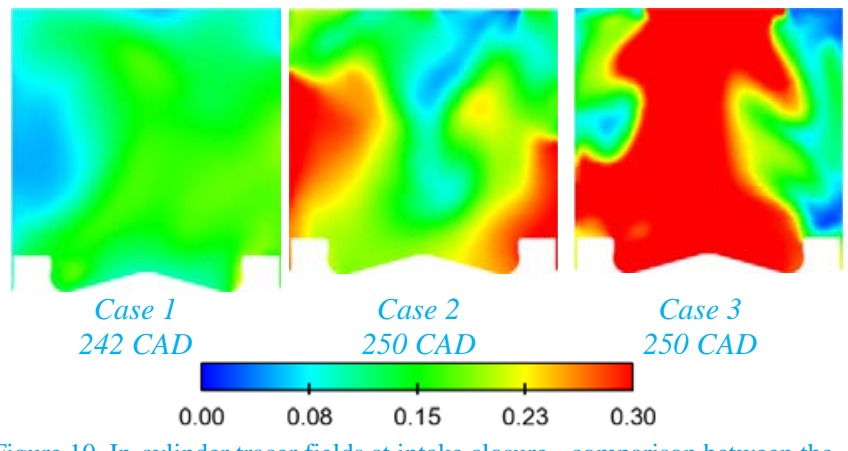

Figure 10. In-cylinder tracer fields at intake closure - comparison between the three investigated cases.

\section{Potential for improvements}

The distributions of in-cylinder tracer at the end of the scavenging, i.e. at the intake closure, are compared in Figure 10 and the axial evolutions of the mean tracer mass fraction are shown in Figure 11. From a global point of view, Case 1 shows the smallest mass fraction of residuals which are in addition well homogeneously distributed. This implies that the final point of the scavenging curve is located on the perfect mixing line, which is the line defined by $y_{B G, \text { cylinder }}=y_{B G \text {,exhaust }}$. If one wishes to further decrease the amount of residual gases by pursuing the scavenging, e.g. increasing the engine $\Delta P$, the improvement of the scavenging ratio will imply the same cost in trapping ratio. Therefore, no further improvement can be anticipated by the modification of the boundary operating conditions and this case is optimized enough. The situation is different for the Case 2 because the burnt gases are located in the bottom sides of the cylinder and close to the exhaust ports. As a consequence, a slight increase of $\Delta P$ may improve the scavenging ratio without a strong decrease of the trapping ratio. The Case 3 shows the most desperate situation since the burnt gases are either located in the piston bowl, in the center or in the top of the cylinder. Pursuing the scavenging for this case may worsen the trapping ratio without a net improvement of the scavenging ratio.

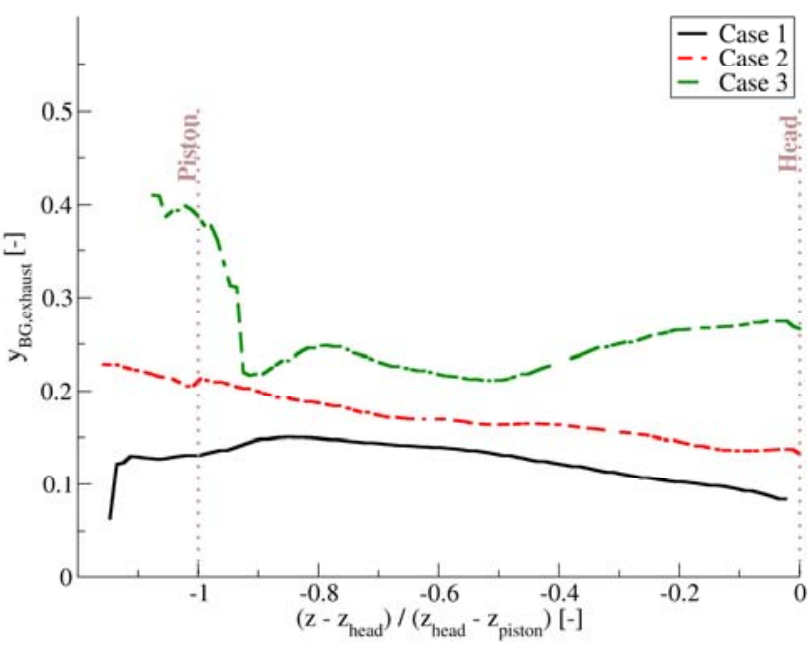

Figure 11. Axial evolution of the in-cylinder tracer fields averaged per axial slice at intake closure - comparison between the three investigated cases.

\section{Conclusions and perspectives}

In the frame of European REWARD, a two-stroke Diesel engine is developed within the perspective to an application to light duty vehicles. On the basis of an uniflow engine architecture, two possible arrangements for the intake and exhaust were investigated: either the standard configuration with an intake insured by ports and the exhaust by poppet valves located in the head or the reverse configuration. Both configurations were benchmarked on the basis of OD system and 3D CFD simulations. System simulations are devoted to find out the best compromise between a gas transfer shifted in the expansion, as it is the case for the standard configuration, or in the compression, as for the reverse configuration. Corrected ISFC determined from 0D system simulations show that the reverse configuration presents a lower fuel consumption of about $10 \mathrm{~g} / \mathrm{kWh}$ for the full load operating point, but this advantage vanishes when running the engine at lower loads. For the full load operating point, the additional expansion offered by the reverse configuration occurs with a negligible loss of trapped mass of fresh gases, whereas these both effects are balanced for the other operating points.

3D CFD simulations were performed to compare the scavenging process from a macroscopic point of view. The comparison between the standard and reverse configurations indicates that the scavenging is more efficient for the standard one, due mainly to the penalty of the drag downstream the valves for the reverse configuration. The benchmark of reverse geometries showed that the generation of swirl strongly worsens the scavenging: this behavior imposes the design of a combustion system operating with almost zero in-cylinder swirl.

In summary, the benefit of the reverse configuration occurs mainly at high load operating conditions, which yields small benefit at medium-low loads. Furthermore, for the reverse Uniflow the achievement of an efficient scavenging is strongly compromised by the unavoidable drag downstream the valves, and the generation of a typical €6 in-cylinder swirl level deteriorates even more the scavenging. In addition to these mentioned drawbacks, the reverse configuration requires a dedicated cooling of the exhaust ports, in order to avoid the excessive heating of the oil flowing from the crankcase to the top of the liner, weakening the lubrication. Due to all 
the above mentioned issues, the standard uniflow architecture is preferred.

Besides the comparison of the different architectures, it has to be mentioned that the corrected ISFC results obtained through this preliminary study, for the three operating conditions, are about 5$10 \%$ higher than the state-of-the art €6 Diesel engines. Indeed, the study described in this paper, i.e. the choice between the standard or reverse uniflow scavenging, represents the first task of REWARD project. The following steps, carried out by all the involved partners (Groupe Renault, CMT-Universitat Politècnica de València, Czech Technical univ., Delphi and IFP Energies Nouvelles), will contribute to the final design of an optimized two-stroke engine:

- definition of the best suited stroke to bore ratio,

- design of the intake transfer ports and the associated intake plenum,

- design of the exhaust ducts and the exhaust valves diagram

- definition of the combustion system with the combined optimization of the bowl shape and the injection system

- $\quad$ assessment of fuel consumptions and pollutants emissions on driving cycles through quasi-static simulations

A comparison with state-of-the art four-stroke Diesel engines will be thus achieved in order to evaluate the market positioning of a twostroke Diesel engine.

\section{References}

1. Xin, Q. H. (2011). Overview of Diesel Engine Applications for Engine System Design-Part 3: Operating and Design Characteristics of Different Applications (No. 2011-01-2180). SAE Technical Paper.

2. Warey, A., Gopalakrishnan, V., Potter, M., Mattarelli, E., \& Rinaldini, C. A. (2016). An Analytical Assessment of the $\mathrm{CO}_{2}$ Emissions Benefit of Two-Stroke Diesel Engines (No. 2016-010659). SAE Technical Paper.

3. Knoll, R. (1998). AVL two-stroke diesel engine (No. 981038). SAE Technical Paper.

4. Masuda, T., Itoh, H., \& Ichihara, Y. (1999). Research on the Practical Application of 1 Liter, Semi-DI, 2-Stroke Diesel Engine to Compact Cars (No. 1999-01-1249). SAE Technical Paper.

5. Nomura, K., \& Nakamura, N. (1993). Development of a new two-stroke engine with poppet-valves: Toyota S-2 engine. A new generation of two-stroke engines for the future, 53-62.

6. Tribotté, P., Ravet, F., Dugue, V., Obernesser, P., Quechon, N., Benajes, J., Novella, R. \& De Lima, D. (2012). Two Strokes Diesel Engine-Promising Solution to Reduce CO2 Emissions. Procedia-Social and Behavioral Sciences, 48, 2295-2314.

7. Brynych, P., Macek, J., Tribotté, P., De Paola, G., \& Ternel, C. (2014). System Optimization for a 2-Stroke Diesel Engine with a Turbo Super Configuration Supporting Fuel Economy Improvement of Next Generation Engines (No. 2014-32-0011). SAE Technical Paper.

8. Redon, F., Kalebjian, C., Kessler, J., Rakovec, N., Headley, J., Regner, G., \& Koszewnik, J. (2014). Meeting stringent 2025 emissions and fuel efficiency regulations with an opposedpiston, light-duty diesel engine (No. 2014-01-1187). SAE Technical Paper.

9. Schweitzer, P. H. (1949). Scavenging of two-stroke cycle diesel engines. Macmillan Co.

Page 11 of 12
10. Mattarelli, E., Rinaldini, C. A., \& Savioli, T. (2016). Port Design Criteria for 2-Stroke Loop Scavenged Engines (No. 2016-01-0610). SAE Technical Paper.

11. Mattarelli, E., Rinaldini, C. A., Savioli, T., Cantore, G., Gopalakrishnan, V. , Warey, A., Balestrino, S.,\& Potter, M. A (2016). Comparison of different scavenging concepts on TwoStroke High-Speed Diesel engines. Conference proceedings: THIESEL 2016. Thermo- and fluid dynamic processes in direct injection engines. València: Universitat Politècnica de València.

12. Ternel, C., De Paola, G. \& Tribotté, P. (2014) Air Loop Characterization and First Potential of an Automotive TwoStroke HSDI Diesel Engine. Conference proceedings: THIESEL 2014. Thermo- and fluid dynamic processes in direct injection engines. València: Universitat Politècnica de València.

13. Abthoff, J., Duvinage, F., Hardt, T., Krämer, M., \& Paule, M. (1998). The 2-Stroke DI-Diesel Engine with Common Rail Injection for Passenger Car Application (No. 981032). SAE Technical Paper.

14. Laget, O., Ternel, C., Thiriot, J., Charmasson, S., Tribotté, P., \& Vidal, F. (2013). Preliminary design of a two-stroke uniflow diesel engine for passenger car. SAE International Journal of Engines, 6(2013-01-1719), 596-613.

15. Ma, J., Zhao, H., Freeland, P., Hawley, M., \& Xia, J. (2014). Numerical Analysis of a Downsized 2-Stroke Uniflow Engine. SAE International Journal of Engines, 7(4), 2035-2044.

16. Rudloff, J., Dulbecco, A., \& Font, G. (2015). The Dual Flame Model (DFM): A Phenomenological 0D Diesel Combustion Model to Predict Pollutant Emissions (No. 2015-24-2388). SAE Technical Paper.

17. Froehlich, M., \& Stewart, N. (2013). TVS ${ }^{\circledR}$ V-Series Supercharger Development for Single and Compound Boosted Engines (No. 2013-01-0919). SAE Technical Paper.

18. CONVERGE (2013). Software (Version 2.2), Convergent Science. Inc., Madison, WI.

19. Yakhot, V., Thangam, S., Gatski, T. B., Orszag, S. A. \& Speziale, C. G. (1992). Development of turbulence models for shear flows by a double expansion technique. Physics of Fluids, 4(7), 1510-1520.

20. Issa, R. I., Gosman, A. D., \& Watkins, A. P. (1986). The computation of compressible and incompressible recirculating flows by a non-iterative implicit scheme. Journal of Computational Physics, 62(1), 66-82.

\section{Contact Information}

Jeremy Galpin

jeremy.galpin@ifpen.fr

+33 (0)147525722

Gaetano de Paola

gaetano.de-paola@ifp.fr

+33(0)147525357 


\section{Acknowledgments}

This work is part of REWARD project. This project has received funding from the European Union's Horizon 2020 research and innovation program under Grant Agreement no. 636380.

The authors would also like to express their gratitude to Convergent Science Inc. for providing their support on the use of CONVERGE code and for helpful discussions.

\section{Definitions/Abbreviations}

BDC

Bottom Dead Center

CFD

Computational Fluid Dynamics
CFL

Courant Friedrich Levy

DFM

Dual Flame Model

DOE

EGR

Design Of Experiments

PISO

Exhaust Gas Recirculation

TDC Pressure Implicit with Splitting Operator

Top Dead Center

Page 12 of 12 